\title{
Simple-zero singularity in toxic phytoplankton-zooplankton model
}

\author{
Yong Wang \\ Department of Information Science and Technology, Tianjin University of Finance and \\ Economics, Tianjin, China \\ ywang@tjufe.edu.cn
}

Keywords: phytoplankton-zooplankton model, simple-zero singularity, bifurcation.

\begin{abstract}
In this paper, we have considered a phytoplankton-zooplankton system with toxic liberation delay. Firstly, we give the critical values of transcritical bifurcation. Then, we derive the normal forms and their unfolding with original parameters in the system near the bifurcation point by the normal form method and center manifold theory. Finally, we show that the plankton system undergoes a transcritial bifurcation, and the equilibrium is unstable.
\end{abstract}

\section{Introduction}

In 2002, based on the field-collected samples and mathematical modeling, Chattopadhyay et al.[1] formulated the following phytoplankton-zooplankton model:

$$
\left\{\begin{array}{l}
\dot{P}=r P\left(1-\frac{P}{K}\right)-\beta f(P) Z, \\
\dot{Z}=\beta_{1} f(P) Z-d Z-\rho g(P) Z .
\end{array}\right.
$$

$f(P)$ represents the predational response function and $g(P)$ represents the distribution of toxic substances. The other parameters is found in the literature [1].

From 2002, many authors [2,3] have studied the complex dynamic behaviors dynamics of phytoplankton-zooplankton models, such as stability of equilibrium, Hopf bifurcation, global Hopf-bifurcation and so on. Some references $[4,5,6]$ have discussed persistence, periodic resonance and chaos of phytoplankton-zooplankton models.

In this paper, we take $f(P)$ is of Holling type II and $g(P)$ is linear, i.e. $f(p)=\frac{P}{\alpha+P}, g(p)=P$. We also consider that the liberation of toxic substances by phytoplankton species is not an instantaneous process but is mediated by some time lag. The model is described by the following system:

$$
\left\{\begin{array}{l}
\dot{P}=r P\left(1-\frac{P}{K}\right)-\frac{\beta P}{\alpha+P} Z, \\
\dot{Z}=\frac{\beta_{1} P}{\alpha+P} Z-d Z-\rho P(t-\tau) Z .
\end{array}\right.
$$

In the reference [7], they explained complex dynamic behaviors of system(2), such as stability of equilibrium, Hopf bifurcation, Hopf-transcritial bifurcation. In this paper, we will study simple-zero singularity of system (2).

\section{Distribution of Eigenvalues}

If $\beta_{1}-\alpha \rho-d=2 \sqrt{d \alpha \rho}, P^{*}<K$ then the system (2) has a unique positive steady-state denoted by $S^{*}\left(P^{*}, Z^{*}\right)$, where

$$
P^{*}=\frac{\beta_{1}-\alpha \rho-d}{2 \rho}, Z^{*}=\frac{r}{\beta}\left(1-\frac{P^{*}}{K}\right)\left(\alpha+P^{*}\right)
$$


We translate the equilibrium $S^{*}\left(P^{*}, Z^{*}\right)$ of the system (2) to the origin. Let $u_{1}(t)=P(t)-P^{*}$ $u_{2}(t)=Z(t)-Z^{*}$, system (2) can be linearized at the zero equilibrium as follows:

where

$$
\left\{\begin{array}{l}
u_{1}(t)=a_{1} u_{1}(t)+a_{2} u_{2}(t) \\
\dot{u_{2}}(t)=b_{1} u_{1}(t)+b_{2} u_{1}(t-\tau)
\end{array}\right.
$$

$$
a_{1}=r-\frac{2 r P^{*}}{K}-\frac{\alpha \beta Z^{*}}{\left(\alpha+P^{*}\right)^{2}}, a_{2}=-\frac{\beta P^{*}}{\alpha+P^{*}}, b_{1}=\frac{\alpha \beta Z^{*}}{\left(\alpha+P^{*}\right)^{2}}, b_{2}=-\rho Z^{*} .
$$

The characteristic equation for system (3) takes the form:

$$
\Delta(\lambda):=\lambda^{2}-a_{1} \lambda-a_{2} b_{1}-a_{2} b_{2} e^{-\lambda \tau}=0
$$

Since $a_{2} b_{1}+a_{2} b_{2}=0$, hence, $\lambda=0$ is a root of Eq.(4). For $\tau=0$, if $a_{1}<0\left(\right.$ i.e. $\left.P^{*}<K<\alpha+2 P^{*}\right)$, then all roots of Eq.(4) except 0 have negative real parts. Next we mainly focus on the case $\tau>0$. Now assume $\tau>0$, It is easy to attain

$$
\Delta^{\prime}(\lambda)=2 \lambda-a_{1}+a_{2} b_{2} \tau e^{-\lambda \tau}
$$

By analyzing, we obtain

$$
\tau=\tau_{j}=\frac{1}{\omega_{0}}(\delta+2 j \pi), j=0,1,2, \cdots
$$

where $\omega_{0}=\sqrt{-2 a_{2} b_{1}-a_{1}^{2}}, \delta=\left\{\begin{array}{l}\arcsin B, A \geq 0, \\ \pi-\arcsin B, A<0,\end{array}\right.$

We summarize the above results in the following theorem:

Theorem 1 Suppose that $\beta_{1}-\alpha \rho-d=2 \sqrt{d \alpha \rho}, P^{*}<K<\alpha+2 P^{*}, \tau>0$, then the following are obtained: If $2 a_{2} b_{1}+a_{1}^{2} \geq 0$, all roots of Eq. (4) except 0 have negative real parts and does not have other roots in the imaginary axis.

\section{Simple-Zero Singularity}

By Theorem (1) (i), we know, if $\beta_{1}-\alpha \rho-d=2 \sqrt{d \alpha \rho}, P^{*}<K<\alpha+2 P^{*}, 2 a_{2} b_{1}+a_{1}^{2} \geq 0, \tau>0$ the characteristic equation Eq.(4) of the linear part of system (2) has a simple zero root and the rest of roots have negative parts. Since $b_{1}+b_{2}=0$, in this section, we treat $b_{1}$ as a bifurcation parameter near $-b_{2}$, and let $b_{1}=-b_{2}+\mu$. In order to investigate the stability of $S^{*}$ for (2), we employ the center manifold theory and normal form method by Faria et al.[8].

Following the same algorithms as those in [8], let $\Lambda=0$ and $B=0$. Clearly, the non-resonance conditions relative to $\Lambda$ are satisfied. Therefore, there exists a 1-dimensional ODE, which governs the dynamics of (2) near $S^{*}$. First, (2) can be written in $C:=C\left([-\tau, 0], \mathbb{R}^{2}\right)$ of the form

$$
\frac{d}{d t} u(t)=L\left(u_{t}\right)+F\left(u_{t}\right)
$$

where $u(t)=(P(t), Z(t))^{T}$,

$$
L(\varphi)=\left(\begin{array}{rr}
a_{1} & a_{2} \\
-b_{2} & 0
\end{array}\right) \varphi(0)+\left(\begin{array}{ll}
0 & 0 \\
b_{2} & 0
\end{array}\right) \varphi(-\tau)
$$

and

$$
F(\varphi)=\left(\begin{array}{c}
a_{20} \varphi_{1}^{2}(0)+a_{11} \varphi_{1}(0) \varphi_{2}(0) \\
\mu \varphi_{1}(0)+b_{20} \varphi_{1}^{2}(0)+b_{11} \varphi_{1}(0) \varphi_{2}(0)+b_{11}^{\prime} \varphi_{1}(-\tau) \varphi_{2}(0)
\end{array}\right) \quad \text { for any } \varphi \in C
$$

with 


$$
\begin{aligned}
& a_{20}=-\frac{r}{K}+\frac{\alpha \beta Z^{*}}{\left(\alpha+P^{*}\right)^{3}}, a_{11}=-\frac{\beta \alpha}{\left(\alpha+P^{*}\right)^{2}}, \\
& b_{20}=-\frac{\alpha \beta_{1} Z^{*}}{\left(\alpha+P^{*}\right)^{3}}, b_{11}=\frac{\alpha \beta_{1}}{\left(\alpha+P^{*}\right)^{2}}, b_{11}^{\prime}=-\rho .
\end{aligned}
$$

Choosing

$$
\eta(\theta)=\left(\begin{array}{cc}
a_{1} & a_{2} \\
-b_{2} & 0
\end{array}\right) \delta(\theta)-\left(\begin{array}{ll}
0 & 0 \\
b_{2} & 0
\end{array}\right) \delta(\theta+\tau)
$$

where $\delta(\theta)$ is a Dirac delta function. We obtain $L(\varphi)=\int_{-\tau}^{0} d \eta(\theta) \varphi(\theta)$.

Using the formal adjoint theory for FDEs (see [12]), we decompose $C$ by $\Lambda$ as $C=P \oplus Q$, where $P=\operatorname{span} \Phi(\theta)$ with $\Phi(\theta)=\left(\begin{array}{l}1 \\ -\frac{a_{1}}{a_{2}}\end{array}\right)$ being the center space for $\frac{d}{d t} u(t)=L\left(u_{t}\right)$. Choose a basis $\Psi$ for the adjoint space $P^{*}$ such that $\langle\Psi, \Phi\rangle=1$, where $\langle\cdot, \cdot\rangle$ is the bilinear form on $C^{*} \times C$ defined by

$$
\langle\psi, \varphi\rangle=\psi(0) \varphi(0)-\int_{-\tau}^{0} \int_{\theta=0}^{s} \psi(\theta-s) d \eta(s) \varphi(\theta) d \theta .
$$

Thus $\Psi(s)=\left(0, \frac{1}{b_{2} \tau-\frac{a_{1}}{a_{2}}}\right)$.

Taking the enlarged phase space $B C=\varphi:[-\tau, 0] \rightarrow C, \varphi$ is continuous on $[-\tau, 0]$ and $\lim _{\theta \rightarrow 0} \varphi(\theta)$ exists, we obtain the abstract differential equation with the form

$$
\frac{d}{d t} u_{t}=A u_{t}+X_{0} F\left(u_{t}\right)
$$

Here, for any $\varphi \in C^{1}([-\tau, 0], R), A \varphi=\dot{\varphi}(\theta)+X_{0}[L(\varphi)-\dot{\varphi}(0)]$, and $X_{0}=X_{0}(\theta)$ is given by

$$
X_{0}= \begin{cases}I, & \theta=0, \\ 0, & \theta \in[-\tau, 0),\end{cases}
$$

Consider the projection $\left.\pi: B C \rightarrow P, \pi\left(\varphi+X_{0} \alpha\right)=\Phi[\langle\Psi, \varphi\rangle+\psi(0) \alpha)\right]$, which leads to the decomposition $B C=P \oplus k e r \pi$. Then, using the decomposition $u_{t}=\Phi x(t)+y, \quad x(t) \in C$, $y=y(\theta) \in Q^{1}$, we decompose (6) as

$$
\left\{\begin{array}{l}
\dot{x}=B x+\Psi(0) F(\Phi x+y) \\
\dot{y}=A_{Q^{1}} y+(I-\pi) X_{0} F(\Phi x+y)
\end{array}\right.
$$

Note that

$$
\begin{aligned}
\Psi(0) F(\Phi x+y)= & \frac{1}{b_{2} \tau-\frac{a_{1}}{a_{2}}} \times\left[\mu(x+y(0))+b_{20}(-x+y(0))^{2}+b_{11}(x+y(0))\right. \\
& \left.\left(-\frac{a_{1}}{a_{2}} x+y(0)\right)+b_{11}^{\prime}(x+y(0))\left(-\frac{a_{1}}{a_{2}} x+y(0)\right)\right]+ \text { h.o.t. }
\end{aligned}
$$

Therefore, the locally invariant manifold for (2) tangent to $\mathrm{P}$ at zero satisfies $y(\theta)=0$ and the flow on this manifold is given by the following 1-dimensional ODE

$$
\dot{x}=m \mu x+n x^{2}+\text { h.o.t. }
$$

where $m=\frac{1}{b_{2} \tau-\frac{a_{1}}{a_{2}}}, n=\frac{b_{20}}{b_{2} \tau-\frac{a_{1}}{a_{2}}}$. 
From above, we know $m<0, n>0$. For $\mu<0$, Eq.(7) has two fixed points, $x=0$ is unstable and $x=-\frac{m}{n} \mu$ is stable. These two fixed points coalesce at $\mu=0$ and, for $\mu>0, x=0$ is stable and $x=-\frac{m}{n} \mu$ is unstable. Thus, an exchange of stability has occurred $\mu=0$. Therefore, system (2) undergoes a transcritial bifurcation. In particularly, if $\mu=0$, the zero solution of (7) is unstable, so the equilibrium $S^{*}$ is unstable.

Then, by the results of the above and Theorem 1, we have the following theorem:

Theorem 2 For system (2), we have the following conclusions: System (2) undergoes a transcritial bifurcation from equilibrium $S^{*}$ for $\tau \in\left\{\tau \mid \tau \geq 0, \tau \neq \tau_{j}\right\}$, and $\beta_{1}-\alpha \rho-d=2 \sqrt{d \alpha \rho}$. In particular, when $\tau \in\left[0, \tau_{0}\right)$, (4) has a simple zero root, and all the other roots have negative real part if $P^{*}<K<\alpha+2 P^{*}$.

\section{Conclusion}

In this paper, we studied simple-zero singularity of toxic phytoplankton-zooplankton model with delay. We have obtained the vector field reduced to the center manifold for simple-zero singularity and discussed their unfolding with the parameter perturbations. The study is an extension of the literature [7].

\section{Acknowledgements}

The authors wish to express their gratitude to the editors and the reviewers for the helpful comments. This work is supported in part by NNSF of China (No.11701410).

\section{References}

[1] J. Chattopadhyay, et al, Toxin producing plankton may act as a biological control for planktonic blooms-field study and mathematical modeling. J. Theor. Biol. 215, 333-344 (2002)

[2] J.T. Zhao, J.J. Wei, Stability and bifurcation in a two harmful phytoplankton-zooplankton system. Chaos. Soliton. Fract. 39, 1395-1409 (2009)

[3] M. Banerjee, E. Venturino, A phytoplankton-toxic phytoplankton-zooplankton model, Ecological Complexity. 8(3), 239-248 (2011)

[4] M. Rehim, et al, On the Dynamical Behavior of Toxic-Phytoplankton-Zooplankton Model with Delay[J]. Discrete Dynamics in Nature and Society, (1) 1-13 (2015)

[5] Z. Zhang, M. Rehim, Global qualitative analysis of a phytoplankton-zooplankton model in the presence of toxicity. International Journal of Dynamics \& Control, 1-12 2016

[6] R.Yuan, et al. Nonresonant double Hopf bifurcation in toxic phytoplankton-zooplankton model with delay. International Journal of Bifurcation \& Chaos 27.2 (2017)

[7] Y. Wang, Wang H, Jiang W. Hopf-transcritical bifurcation in toxic phytoplankton-zooplankton model with delay. Journal of Mathematical Analysis \& Applications. 415(2) 574-594 (2014)

[8] T. Faria, LT. Magalhaes, Normal Forms for Retarded Functional Differential Equations and Applications to Bogdanov-Takens Singularity. J. Differ. Equat. 122 201-224 (1995) 